\title{
Cambios histopatológicos de la mucosa bucal de ratas expuestas al humo de cigarrillos
}

\section{Histopathological oral tissues changes of rats exposed to tobacco smoke}

\author{
Flete Estrada A*, Cáribas A*, Payares G*, Villarroel-Dorrego M*
}

\section{RESUMEN}

Introducción: El propósito de esta investigación fue evaluar el efecto del humo del tabaco en la mucosa bucal "in vivo" usando un modelo animal.

Materiales y métodos: La población de estudio estuvo constituida por 20 ratas hembras Sprague Dawley, 10 expuestas al humo de 10 cigarrillos diarios por 16 semanas (GEC) y un grupo control no expuesto (GC). Las ratas fueron sacrificadas y se extrajo la lengua para su estudio histopatológico. Variables categóricas fueron comparadas mediante la prueba U de Mann-Whitney. Valores $p<0,05$ fueron considerados estadísticamente significativos. Resultados: La mucosa lingual del GEC mostró mayores cambios epiteliales proliferativos que el GC, como hiperplasia basal $(p=0,001)$, acantosis $(p=0,0001)$, hipercromatismo y picnosis nuclear $(p=0,010$ y 0,014 respectivamente), y displasia epitelial $(p=0,0001)$. En el corion de la mucosa del GEC se observó un incremento del infiltrado inflamatorio crónico $(p=0,007)$; y aunque no hubo aumento en el número de vasos sanguíneos en el GEC, es importante resaltar que la ubicación de los mismos fue muy próxima al epitelio de revestimiento expuesto al tabaco.

Discusión: El presente estudio, usando un modelo animal, demuestra que el humo del tabaco induce cambios precoces en la arquitectura tisular y morfología de células epiteliales de la mucosa bucal de aspecto macroscópicamente sano.

Palabras clave: Tabaquismo, modelo animal, cambios histológicos, mucosa bucal.

\section{SUMMARY}

Introduction: The purpose of this study was to evaluate the histopathological changes in oral tissues of rats exposed to tobacco smoke.

Materials $\mathcal{E}$ methods: 20 female rats Sprague Dawley were included in the study and 10 rats exposed to environmental tobacco smoke of 10 cigarettes daily for 16 weeks. All animals were sacrificed and tissues extracted, died using HEE and finally evaluated under light microscope. Categorical variables were compared using $U$ Mann-Whitney test. $P$ values $<0.05$ were considered statistically significant.

Results: Oral mucosa of rats exposed to tobacco showed augmented epithelial changes as basal hyperplasia basal $(p=0,001)$, acanthosis $(p=0,0001)$, nuclear hyperchromatism and picnosis $(p=0,010$ y 0,014 respectively), and epithelial dysplasia $(p=0.0001)$. Increased chronic inflammatory infiltrate was also observed in this group $(p=0.007)$, as well as, a higher number of blood vessels although difference was not statistically significant.

Discussion: Tobacco inhalation showed to produce tissue alterations of macroscopically healthy mucosa of rats. This study, using an animal model, demonstrated microscopical oral mucosa changes produced by tobacco smoke without clinical alterations.

* Universidad Central de Venezuela. Caracas, Venezuela. 
Key words: Smoking, animal model, histological changes, oral mucosa.

Fecha de recepción: 20 de octubre de 2012.

Aceptado para publicación: 17 de diciembre de 2012.

Flete Estrada A, Cáribas A, Payares G, Villarroel-Dorrego M. Cambios histopatológicos de la mucosa bucal de ratas expuestas al humo de cigarrillos. Av. Odontoestomatol 2014; 30 (4): 219-225.

\section{INTRODUCCIÓN}

El tabaquismo, hoy en día, ha sido definido como una enfermedad crónica contagiosa caracterizada por una dependencia a la nicotina, que cursa con períodos de abstinencia y recaídas, asociada a todas las formas de consumo del tabaco (1). Debido a la alta frecuencia del consumo de tabaco inhalado, diversos estudios han reportado los efectos producidos por la exposición de forma pasiva al humo que se produce, cuyos constituyentes hacen que sea un gas menos ácido más fácilmente absorbible (2-4).

Diversos componentes del tabaco han sido implicados en el proceso de carcinogénesis bucal, el cual se da en múltiples fases y requiere la adquisición de propiedades fundamentales como autoproliferación celular, insensibilidad a las señales antiproliferativas, evasión de la apoptosis, potencial replicativo ilimitado, mantenimiento de la vascularización, invasión a los tejidos y metástasis (5). Ciertos elementos de la fase particulada del humo del tabaco como, por ejemplo, los hidrocarbonos aromáticos policíclicos y los compuestos fenólicos, suelen actuar como iniciadores en dicho proceso, mientras que los que componen la fase gaseosa pueden jugar un rol de promotores o cocarcinógenos, que estimulan a células que ya han sido iniciadas $(6,7)$.

En tal sentido, se tiene que una vez que dichos compuestos se encuentran en el organismo sufren un proceso de metabolización del cual se liberan productos que se unen covalentemente al ADN de las células produciendo daño al material genético y evasión de los mecanismos de reparación celular por inhibición del gen $p 53$, traduciéndose en un aumento de la actividad replicativa y proliferativa induciendo cambios citológicos y estructurales de los tejidos, siendo el tejido epitelial uno de los principalmente afectado (8-12).
Partiendo del hecho de que existe una progresión en los cambios microscópicos antes que puedan evidenciarse cambios macroscópicos en los tejidos bucales por acción de dichos compuestos, el propósito de este trabajo fue evaluar el efecto microscópico de la exposición crónica al humo del cigarrillo en la mucosa bucal de ratas.

\section{MATERIALES Y MÉTODOS}

\section{Población animal}

Se incluyeron en el estudio 20 ratas hembras Rattus norvegicus, de la cepa Sprague Dawley, entre 250 y 300 gramos de peso, las cuales fueron adquiridas en el Instituto Venezolano de Investigaciones Científicas y trasladadas al Bioterio del Instituto de Biología Experimental, Facultad de Ciencias de la Universidad Central de Venezuela, para el cuidado y mantenimiento de los animales bajo las "Normas de cuidado y uso de animales con propósitos científicos año 2004" (13), donde se llevó a cabo la fase experimental.

\section{Protocolo de administración del humo de cigarrillos}

La fase experimental tuvo una duración de 16 semanas (74 días de exposición). Las ratas fueron separadas en 2 grupos al azar, 10 de ellas constituyeron el grupo expuesto al humo del cigarrillo (GEC) y 10 fueron el grupo control no expuesto (GC).

Para la administración del humo de cigarrillos el grupo que sería expuesto era colocado en un lugar aislado del grupo control para evitar el mínimo contacto de este último al humo. Dicha administración se realizó por medio del succionador de Gleras, el cual 
aspiró un cigarrillo con una presión mínima de 80 $\mathrm{mm}$ de $\mathrm{Hg}$, en un minuto aproximadamente, expulsando el humo a través de una válvula y con la ayuda de una manguera el mismo se llevó al interior de la jaula metálica que se mantuvo parcialmente cerrada para lograr la permanencia del humo en contacto con las ratas por un tiempo mínimo estimado de 5 minutos (Fig. 1).

Los cigarrillos fueron administrados aumentando la cantidad de forma progresiva, comenzando con un cigarrillo hasta llegar a un máximo de 10 cigarrillos diarios, cantidad que se mantuvo en un período de 16 semanas. El tiempo de contacto con el humo de cada cigarrillo fue de 6 minutos aproximadamente ( 1 minuto de consumo del cigarrillo más 5 minutos para la dispersión del humo) lo que dio un total de 60 minutos diarios.

Culminada la fase de exposición las ratas fueron sacrificadas utilizando sobredosis de Nembutal sódico. Posterior a la extracción de la lengua, el órgano fue sumergido en una solución de formalina tamponada al $10 \%$, preparada a un $\mathrm{pH}$ de 7,4 .

\section{Variables y análisis estadístico}

El análisis descriptivo de la mucosa lingual fue dividido en características arquitectónicas y citológicas del epitelio e intensidad del infiltrado inflamatorio presente así como cantidad de vasos sanguíneos en el corion. El análisis estadístico se realizó mediante

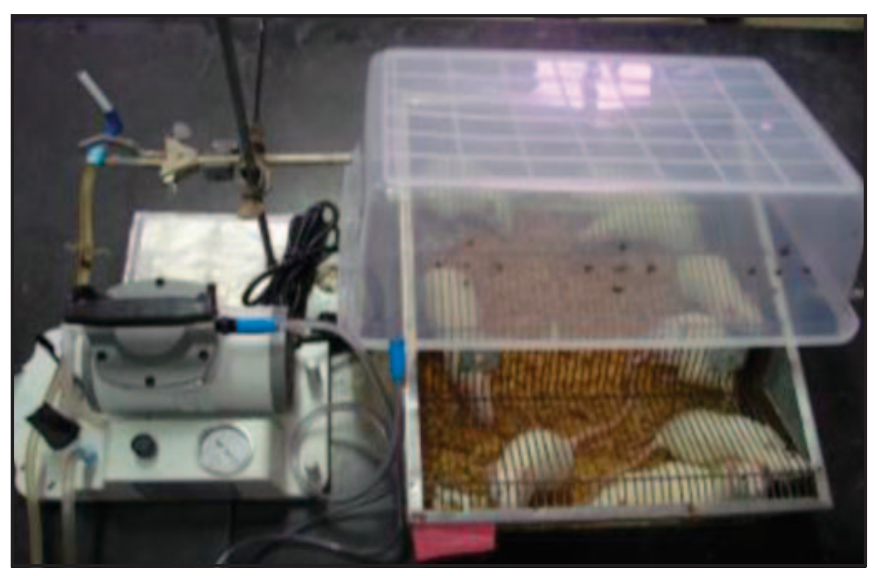

Fig. 1. Sistema de administración del humo del tabaco. el uso del software SPSS versión 17.0, por medio de éste se determinó la frecuencia de cada variable y se realizó el análisis descriptivo y comparativo de las variables. Las variables cualitativas fueron estimadas y los datos comparados mediante la Prueba no paramétrica de Mann-Whitney. El nivel de significancia fue establecido en $0,05(\alpha=0,05)$ y valores $p$ menores a 0,05 fueron considerados como estadísticamente significativos.

\section{RESULTADOS}

En cuanto a las características estructurales del epitelio, el GEC mostró mayores cambios proliferativos que el GC, observándose una marcada acantosis presente en 8 de las 10 muestras analizadas (80\%) e hiperplasia basal (70\%), estando ausente en la totalidad de las muestras del grupo control con diferencias estadísticamente significativas de $(p=0,0001 \mathrm{y}$ 0,001 , respectivamente). Cambios citológicos importantes como hipercromatismo $(50 \%)$ y picnosis nuclear $(60 \%)$ también estuvieron presentes en muestras del GEC con valores de $p=0,010$ y 0,014.

En base a las características presentes se diagnosticó la patología epitelial presente siguiendo los criterios establecidos por la Organización Mundial de la Salud (14): del GEC, un $10 \%(n=2)$ de las muestras analizadas presentaron hiperplasia epitelial (Fig. 2A), un $35 \%(n=7)$ presentaron displasia epitelial leve (Fig. 2B) y sólo 1 (5\%) evidenció una displasia epitelial moderada (Fig. 2C), estos rasgos histopatológicos estuvieron ausentes en el GC y en ninguna muestra se observó displasia epitelial severa ni carcinomas invasivos.

Con respecto a los cambios observados en el corion de la mucosa lingual, el infiltrado inflamatorio presente era de tipo crónico, con una tendencia de leve a moderada en las muestras analizadas del GEC y de ausente a leve en el GC, esta diferencia es manifiesta estadísticamente significativa $(p=0,007)$ (Fig. 3).

Por otra parte, no hubo diferencias estadísticas en cuanto a la cantidad de vasos sanguíneos presentes en las muestras de ambos grupos de estudio, sin embargo, es resaltante la ubicación muy próxima al epitelio observada en las muestras del GEC (Fig. 4). 


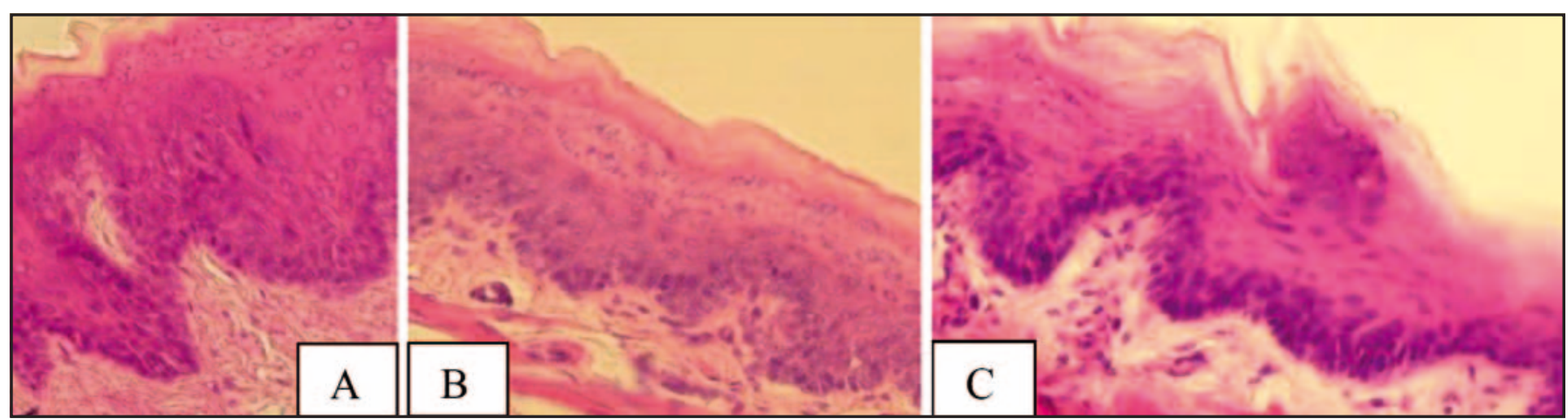

Fig. 2. Patología epitelial presente en mucosa lingual de ratas expuestas al humo del cigarrillo, tinción H-E. A: Hiperplasia Epitelial; B: Displasia epitelial leve; C: Displasia epitelial moderada.

\section{DISCUSIÓN}

Estudios con roedores han demostrado que componentes del tabaco inducen cambios precoces en la arquitectura tisular y morfología de células epiteliales de la mucosa bucal, evidenciando que posterior a la exposición a carcinógenos derivados del cigarri-

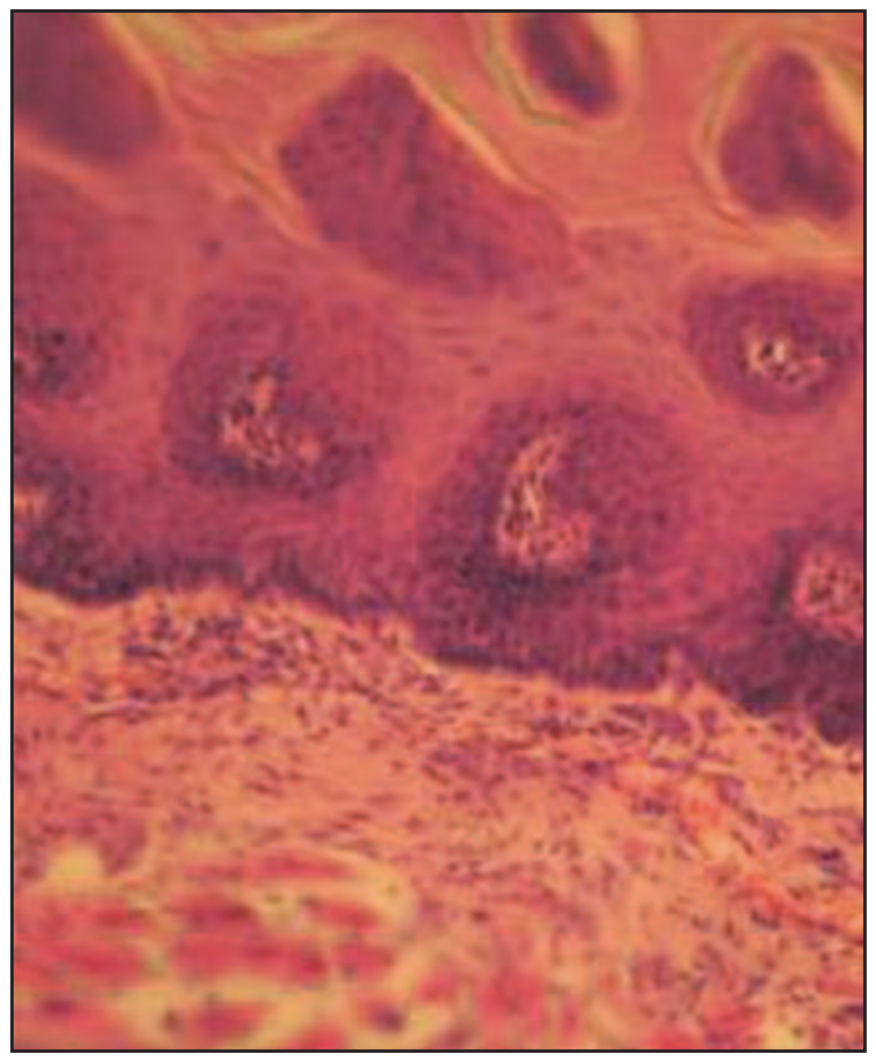

Fig. 3. Infiltrado Inflamatorio de intensidad moderada en el corion de la mucosa lingual de ratas del GEC. Tinción H-E. llo, bien sea por vía inhalatoria, oral o de forma tópica sobre las mucosas, se produce un estímulo sobre los queratinocitos, los cuales reaccionan aumentando la tasa de proliferación y desarrollando cam-

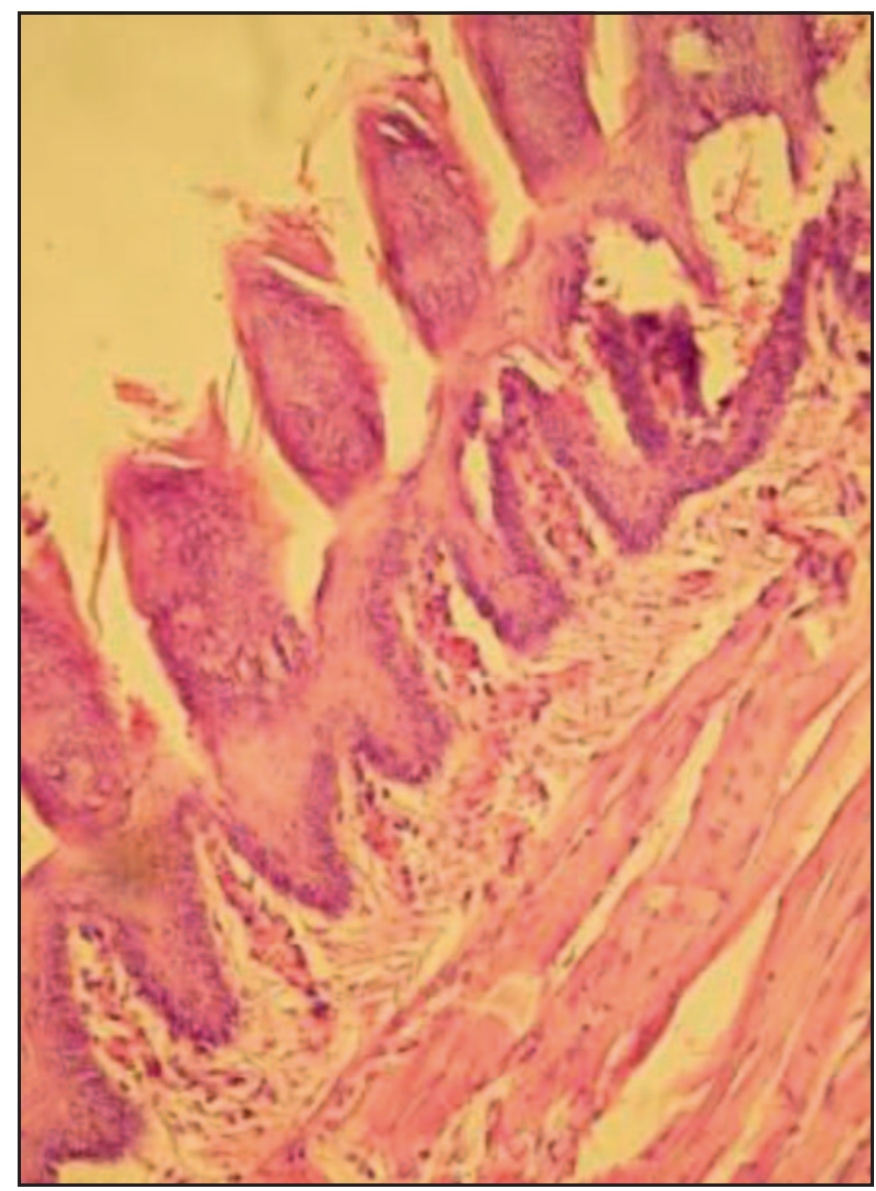

Fig. 4. Ubicación subepitelial de vasos sanguíneos en el corion de la mucosa lingual de ratas del GEC. Tinción H-E. 
bios citológicos que indican una mayor actividad nuclear (15-17).

En investigaciones realizadas en ratas Sprague Dawley, expuestas al humo de cigarrillos, se observó que los componentes del humo del tabaco sobre la inducción de los genes que participan en el metabolismo de los carcinógenos químicos, siendo una ruta sugerida para la carcinogénesis la activación de los genes de la fase I, principalmente el citocromo P450 dependiente de los hidrocarbonos aromáticos policíclicos, los cuales forman reactivos intermedios biológicamente activos que interfieren con un locus específico del ADN resultando en mutaciones que son iniciadoras de la carcinogénesis, siendo el blanco principal la mutación del gen $p 53$ y ras, lo cual ha sido corroborado en investigaciones más recientes en mucosa bucal de humanos $(18,19)$. En este sentido, la alteración de estos genes en roedores por exposición crónica al humo de cigarrillos puede explicar los cambios observados en la presente investigación, en la mucosa bucal de las ratas del GEC, en las cuales se pudo observar alteraciones en la arquitectura del epitelio y en la morfología de los queratinocitos (20-22).

En la presente muestra de estudio se pudo evidenciar cambios epiteliales como anisonucleosis e hipercromatismo, con incremento proliferativo en la capa basal y estrato espinoso que produjeron una hiperplasia epitelial, pero conforme la exposición se mantuvo en el tiempo, las células comenzaron a ceder demostrando picnosis.

No obstante, la respuesta desarrollada ante la exposición a los carcinógenos varía de un individuo a otro determinado por el perfil genético, en la carcinogénesis por químicos hay un número de pasos que se dan bajo el control genético como lo son la activación metabólica y catabolismo de los carcinógenos químicos, su acceso a los tejidos blancos, susceptibilidad de las células blanco, características patofisiológicas del tejido epitelial y la capacidad para reparar el ADN $(23,24)$. En resultados obtenidos se observó que bajo las mismas condiciones experimentales de tiempo de exposición y dosis de cigarrillos los cambios en el epitelio se presentaron de tres maneras distintas: como hiperplasia epitelial, displasia epitelial leve y displasia epitelial moderada.
En cuanto a las características del infiltrado inflamatorio observado en la presente investigación se tiene que a pesar que el grado de intensidad fue mayor en el grupo experimental expuesto al humo de cigarrillos en comparación con el grupo control, se pudo notar que hubo una variación con respecto a lo reportado en otras investigaciones, ya que infiltrado inflamatorio de leve a moderado se observó en asociación a las patologías epiteliales de menor severidad como hiperplasia y displasia epitelial leve, mientras que en la displasia epitelial moderada estuvo presente un infiltrado inflamatorio leve.

Se ha encontrado una asociación positiva en aquellos tejidos que son expuestos a una irritación crónica por el humo del cigarrillo que inducen una inflamación subsecuente. La infiltración de células inflamatorias está asociada a la presencia de lesiones malignas, el cual se incrementa conforme va progresando la patología epitelial, es decir en la evolución desde una hiperqueratosis progresando a displasias epiteliales hasta llegar al carcinoma de células escamosas (25-27).

Finalmente, diversos estudios han apoyado el incremento de la vascularidad por estímulos de los productos del cigarrillo, otros han señalado a través de estudios in vitro y en humanos la disminución del número de vasos subepiteliales, principalmente en las vías aéreas y el parénquima pulmonar, y también de forma sistémica $(28,29)$. El hallazgo en relación a los vasos sanguíneos presentados en esta investigación, en las muestras pertenecientes al grupo expuesto al humo de cigarrillos con respecto al grupo control, la ubicación tan próxima al epitelio, de manera característica en el vértice de las papilas dérmicas podría proveer el aporte sanguíneo necesario para suplir la demanda de nutrientes requeridos por las células proliferando de forma incrementada (30).

Estos resultados sugieren que la inspección visual y el examen rutinario bucal no parecieran ser el método ideal para la prevención y detección de desórdenes potencialmente malignos ya que los cambios microscópicos no son visibles clínicamente, al menos en las etapas tempranas de exposición al tabaquismo. El presente estudio, usando un modelo animal, demuestra que el humo del tabaco induce 
cambios precoces en la arquitectura tisular y morfología de células epiteliales de la mucosa bucal de aspecto macroscópicamente sano.

\section{BIBLIOGRAFÍA}

1. Vaccarino F, Rotzinger S, Obot I, Monteiro M. Neurociencia del consumo y dependencia de sustancias psicoactivas. Organización Mundial de la Salud. 2005.

2. Woodward A, Al- Delaimy W. Measures of exposure to environmental tobacco smoke. Validity, Precision and relevance. Ann N Y Acad Sci 1999;895:156-72.

3. Husgafvel-Pursiainen K. Genotoxicity of environmental tobacco smoke: a review. Mutat Res 2004;567:427-45.

4. Bozkurt A, Sainöz S, Özçlrplcl B, Özgür S, Sahinöz $\mathrm{T}$, Acemoglu $\mathrm{H}$, et al. Patterns of active and passive smoking, and associated factors, in the South-east Anatolian Project (SEAP) region in Turkey. BMC Public Healht 2006;6:15.

5. Rakoff-Nahoum S. Why Cancer and Inflammation? Yale J Biol Med 2006;79(3-4):123-30.

6. Hoffmann D, Hecth SS, Wynder L. Tumor promoters and cocarcinogens in tobacco carcinogenesis. Environ Health Perspect 1983;50: $247-$ 57.

7. Coppe JP, Boysen M, Sun CH, Wong BJ, Kang $\mathrm{MK}$, Park $\mathrm{NH}$, et al. A role for fibroblasts in mediating the effects of tobacco-induced epithelial cell growth and invasion. Mol Cancer Res 2008;6 (7):1085-98.

8. Hetch S. Tobacco and Cancer: Approaches using carcinogen biomarkers and chemoprevention. Ann N Y Acad Sci 1997;833:91-111.

9. Dumble M, Gatza C, Tyner S, Venkatachalam S, Donehower L. Insights into aging obtained from p53 mutant mouse models. Ann N Y Acad Sci 2004;1019:171-7.
10. Kuper H, Adami H, Boffetta P. Tobacco use, cancer causation and public health impact. J Intern Med 2002;251(6):455-66.

11. Parke D. The Cytocromes P450 and mechanisms of chemical carcinogens. Environ Health Perspect 1994;102(10):852-3.

12. Lodovici M, Bigagli E. Biomarkers of induced active and passive smoking damage. Int $J$ Environ Res Public Health 2009;6(3):874-88.

13. National Advisory Committee for Laboratory animal research. Guidelines on the Care and use of animals for scientific purposes. 2004.

14. Barnes L, Everson JW, Reichart PA, Sidransky D. World Health Organization classification of tumours. Pathology and Genetics of Head and neck tumours. London. IARC Pres. 2005.

15. Pressentin M, Kosinska W, Guttenplan J. Mutagenesis induced by oral carcinogens in lacZ mouse (Muta TMMouse) tongue and other oral tissues. Carcinogenesis 1999;20 (11):2167-70.

16. Caldeira E, Fabrega C, Padovani C, Camilli J, García P, Alves V. Morphological alterations in epithelium of the oral mucosa of rats (Rattus norvegicus) submitted to long-term systemic nicotine treatment. Arch Oral Biol 2007;52:839.

17. Kitakawa D, Cabral L, Marques M, Salvadori E, Ribeiro D. Medium - term tongue carcinogénesis assays: A comparative study between 4nitroquinolina-1 oxide (4NQO)- induced rat and dimethylbenzanthrancene (DMBA)- induced hamster carcinogenesis. J Exp Anim Sci 2006;43: 219-27.

18. Gebel S, Gerstmayer B, Bosio A, Haussmann $H$, Van E, Müller T. Gene expression profiling in respiratory tissues from rats exposed to mainstream cigarette smoke. Carcinogénesis 2004;25(2), 169-78.

19. Boyle JO, Gümüs ZH, Kacker A, Choksi VL, Bocker JM, Zhou XK, et al. Effects of cigarette smoke on 
the human oral mucosal transcriptome. Cancer Prev Res (Phila) 2010;3(3): 266-78.

20. Lee CK, Brown BG, Reed BA, Rahn CA, Coggins CR, Doolitle DJ, et al. Fourteen - Day inhalation study in rats, using aged and diluted sidestream smoke from a reference cigarette. II. DNA adducts and alveolar macrophage cytogenetics. Fundam Appl Toxicol 1992;19(1):141-6.

21. Sridhar S, Schembri F, Zeskind J, Shah V, Gustafson AM, Steiling K, et al. Smoking-induced gene expression changes in the bronchial airway are reflected in nasal and buccal epithelium. BMC Genomics 2008;9:259.

22. Shabana AH, EL-Labban NG, Lee KW. Morphometric analysis of basal cell layer in oral premalignant white lesions and squamous cell carcinoma. J Clin Pathol 1987;40(4):454-8.

23. Kitano M. Hosts genes controlling the susceptibility and resistance to squamous cell carcinoma of the tongue in a rat model. Pathol Int 2000;50(5):35362.

24. Zhang L, Wu R, Dingle RW, Gairola CG, Valentino $\mathrm{J}$, Swanson HI. Cigarette smoke condensate and dioxin suppress culture shock induced senescence in normal human oral keratinocytes. Oral Oncol 2007;43(7):693-700.

25. Winn DM. Tobacco Use and Oral Disease. J Dent Educ 2001;65(4):306-12.

26. Gannot G, Gannot I, Vered H, Buchner A, Keisari Y. Increase in immune cell infiltration with progression of oral epithelium from hyperkeratosis to dysplasia and carcinoma. Br J Cancer 2002;86 (9):1444-8.

27. Nie L, Xiang RL, Liu Y, Zhou WX, Jiang L, Lu B, et al. Acute pulmonary inflammation is inhibited in CXCR3 knockout mice after short-term cigarette exposure Acta Pharmacol Sin 2008;29 (12), 1432-9.

28. Yamada G, Shijubo N, Kitada J, Takahashi M, Otsuka M, Fujii, M, et al. Decreased subepithelial microvasculature observed by high magnification bronchovideoscope in the large airways of smokers. Intern Med 2008;47(18):1579-83.

29. Liu SY, Chang LC, Pan LF, Hung YJ, Lee CH, Shieh YS. Clinicopathologic significance of tumour cell- lined vessel and microenvironment in oral squamous cell carcinoma. Oral Oncol 2008; 44(3):277-85.

30. Johnstone S, Logan RM. Expresion of vascular endothelial growth factor (VEGF) in normal oral mucosa, oral dysplasia and oral squamous cell carcinoma. Int J Oral Maxillofac Surg 2007;36 (3):263-6.

\section{CORRESPONDENCIA}

Mariana Villarroel Dorrego

Instituto de Investigaciones Odontológicas

Facultad de Odontología

Universidad Central de Venezuela

Ciudad Universitaria, Los Chaguaramos

Caracas 1060. Venezuela.

Correo electrónico: mariana.villarroel@ucv.ve 